\title{
Novel therapies for pancreatic cancer: setbacks and progress
}
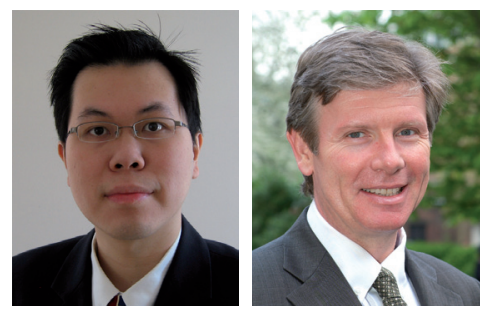

"Years of intensive research have made pancreatic cancer one of the most described diseases in terms of its molecular biology and pathogenesis, and with that, many therapeutic targets have been identified."

\section{Han Hsi Wong' \& Nicholas R Lemoine ${ }^{\dagger}$}

IInstitute of Cancer, Barts \& The London School of Medicine \& Dentistry, Queen Mary University of London. Charterhouse Square, London ECTM 6BQ, UK

†Author for correspondence: Tel.: +44 2078823500 m. Fax: +44 2078823888 m director@amcr.amul.ac.uk

Pancreatic cancer continues to be one of the most difficult malignancies to treat. It is ranked tenth in cancer incidence but is the fourth leading cause of cancer mortality [1]. At present, surgery for resectable tumor and gemcitabine-based chemotherapy for advanced disease are the main forms of treatment; yet disturbingly, its 5-year survival rate of $5 \%$ has not improved over the last 30 years. Clearly, there is an urgent need for new therapeutic strategies. Years of intensive research have made pancreatic cancer one of the most described diseases in terms of its molecular biology and pathogenesis, and with that, many therapeutic targets have been identified [2]. One example is the activation of the EGF-receptor (EGFR) signaling pathway. Erlotinib, an EGFR tyrosine kinase inhibitor, was the first targeted therapy to be approved by the US FDA in 2005 for pancreatic cancer treatment. Although it has shown a statistically significant benefit in combination with gemcitabine in a Phase III trial of advanced pancreatic cancer, improvement in survival was small (median survival of 6.24 vs 5.91 months with gemcitabine alone) and appears to favor a subgroup of patients, such as males and those who developed a more severe skin rash [3]. However, cetuximab, a monoclonal antibody that also inhibits EGFR, was ineffective against advanced pancreatic cancer [4]. In fact, a number of other targeted agents have been tested in Phase III trials, including those against VEGF (bevacizumab), gastrin (G17DT), gastrin receptor (gastrazole), Ras farnesyltransferase (tipifarnib) and matrix metalloproteinases (marimastat and tanomastat), but have all failed to make any measurable impact on patient survival [2]. More recently, a Phase III trial of immunotherapy using the GV1001 telomerase peptide vaccine (telomerase is expressed in the majority of pancreatic cancers and contributes to cell immortalization) was stopped prematurely as results showed no survival benefit in combination with gemcitabine in advanced pancreatic cancer patients [5].

"...targeted agents have been tested in
Phase III trials ... but have all failed to
make any measurable impact on
patient survival."

Data from these molecularly targeted approaches suggest that there are still gaps in our knowledge of pancreatic carcinogenesis and the drugs used; for example, the Hedgehog signaling pathway, which is implicated in pancreatic tumorigenesis, could have affected the delivery of drugs to cancer cells owing to poor vascularization [6]. Bortezomib, a proteosome inhibitor that prevents the degradation of $I \kappa B$ and is licensed for the treatment of refractory multiple myeloma, failed to show any benefit in a Phase II pancreatic cancer trial [7], and this could be related to the paradoxical activation of other antiapoptotic and mitogenic pathways by this agent [8]. Celecoxib, a COX2 inhibitor currently in Phase III trial, has antitumoral activity that does not correlate with its inhibition of COX2, suggesting the involvement of alternative mechanisms [9]. Recent genomic analyses by Jones et al. revealed that an average of 63 genetic alterations affect a core set of 12 signaling pathways and processes that are genetically altered in $67-100 \%$ of cases of pancreatic cancer [10]. As such, targeted agents should also focus on the blockade of multiple pathways, or inhibition of a signaling pathway at multiple levels. Equally critical is a more stringent set of criteria by which agents are moved into Phase III clinical trials and appropriate patient selection (e.g., disease stage or by predictive biomarkers).

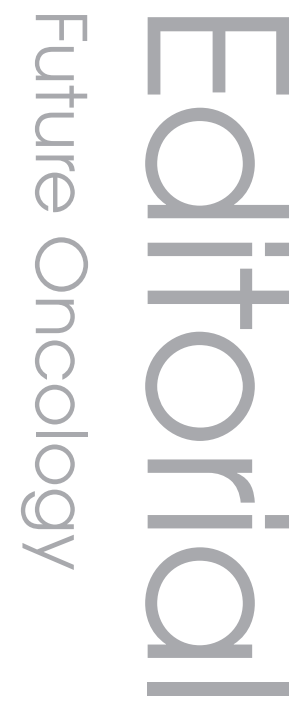


While much work is ongoing for the development of more effective molecularly targeted agents, many groups have focused their attention on gene-therapy approaches [11]. This involves the introduction of exogenous nucleic acid to express, restore or inhibit a gene of interest. They can be delivered by viral (e.g., adenovirus and retrovirus) and nonviral (e.g., naked DNA, liposome and polymer) delivery systems. Viral vectors remain the most effective vehicle and have therefore been widely studied. The conventional approach is the delivery of genes that have antitumoral activity, such as tumor suppressor, proapoptotic, antiangiogenic and immunostimulatory genes. The first government-approved cancer gene-therapy agent was Gendicine $^{\circledR}$ (SiBiono GeneTech, Shenzen, China) in China in 2004. It is a replicationdefective adenovirus 5 (Ad5) that delivers the tumor suppressor gene TP53 to head-and-neck squamous cell carcinoma. However, in the USA, a similar virus called $\operatorname{Advexin}^{\circledR}$, developed by Introgen Therapeutics (TX, USA), did not show convincing outcomes and was subsequently rejected by the FDA. This virus has not been tested clinically in pancreatic cancer. A recent development by Epeius Biotechnologies (CA, USA) is Rexin- $G^{\circledR}$, a retroviral vector carrying the dominant negative mutant of the oncogenic protein cyclin G1. Administered intravenously, it targets collagen-exposed areas, such as the tumor environment, and has shown promising results in Phase I/II trials of gemcitabineresistant pancreatic cancer [12]. Further studies are planned.

\section{"...targeted agents should also focus on the blockade of multiple pathways, or inhibition of a signaling pathway at multiple levels."}

One major shortcoming with the use of nonreplicating viral vectors is the short-lived expression of therapeutic transgene. An improvement on this would be the use of replication-selective oncolytic virus, whereby efficacy is enhanced by virus multiplication, lysis and spread to adjacent tumor cells. A Phase I trial of the oncolytic herpes simplex virus-1 encoding the gene for granulocyte-macrophage colony-stimulating factor, OncoVEX ${ }^{\mathrm{GM}-\mathrm{CSF}}(\mathrm{BioVex}, \mathrm{MA}$, USA), is currently being tested on patients with unresectable pancreatic cancer. An oncolytic Ad2/Ad5 hybrid, ONYX-015 ${ }^{\circledR}$ (Onyx Pharmaceuticals, CA, USA), has been tested in two Phase I/II trials where the virus was injected directly into pancreatic tumors [13,14]. Disappointingly, no virus replication was detected on fine-needle biopsy of the tumors, unlike other trials for head-and-neck cancer and liver metastases of colorectal carcinoma. The reasons for these results are diverse, determined by the complex interaction between the tumor, the virus (e.g., low expression of viral attachment receptors on tumor cells and deletion of genes that affected its potency) and the host immune response (e.g., increased virus clearance and reduced T-cell-mediated antitumoral activity) [15]. In recent years, a number of studies have been conducted to optimize this treatment modality, which include increasing viral infectivity and potency $[16,17]$, the use of different viral species [18-20], arming oncolytic viruses with therapeutic genes [21,22] and combining viruses with chemotherapy [23-25]. Results have been promising, but it will be some time before they can be translated into the clinical context.

"While much work is ongoing for the development of more effective molecularly targeted agents, many groups have focused their attention on gene-therapy approaches."

Another approach that has gained popularity among the scientific community is the use of RNA-directed gene-silencing strategies. They regulate gene expression post-transcriptionally by binding to specific target mRNA and blocking its translation to protein. This includes the use of antisense oligonucleotides and siRNAs. Although the antisense inhibitor of oncogenic HRas, ISIS 2503, had demonstrated activity in a Phase II trial of locally advanced and metastatic pancreatic cancers [26], development of this drug was abandoned following the failures of other similar compounds, such as ISIS 3521 and oblimersen, in lung cancer and melanoma, respectively. The siRNAs are highly specific for their target mRNAs and, via interaction with the RNA-induced silencing complex, cause cleavage of the mRNA or translational block. In vitro and in vivo results against targets such as KRas (mutated in 95\% of pancreatic cancers) [27], hypoxia-inducible factor- $1 \alpha$ (a transcription factor that is activated in hypoxic tumors) [28], the zinc transporter ZIP4 [29] (expressed in 94\% of pancreatic carcinomas [30]), Notch 1 [31] and signal transducer and activator of transcription 3 [32] (proteins involved in tumorigenic signaling pathways), are encouraging, although 
this technology has yet to be tested in patients with pancreatic cancer. CALAA-01 (Calando Pharmaceuticals, CA, USA), a nanoparticle containing siRNA against the M2 subunit of ribonucleotide reductase, has now entered a Phase I trial for the treatment of solid tumors.

The discovery of miRNAs in 1993 spawned a number of studies investigating targeting these molecules in cancer treatment. They are small, endogenous RNA nucleotides that regulate gene transcription post-transcriptionally in a similar manner to siRNAs, and they can be either oncogenic or tumor-suppressive, depending on their target mRNAs. An individual miRNA can inhibit multiple mRNAs, thereby making it an ideal therapeutic target. A large number of miRNA precursors are aberrantly expressed in pancreatic cancer [33], and treatment strategies include the reconstitution of tumor-suppressive miRNAs and the knockdown of oncogenic miRNAs by coding vectors, anti-miRNA oligonucleotides or molecular drugs. Studies of this treatment approach have been limited in pancreatic cancer but are gradually increasing. Examples of in vitro work include targeting the downregulation of miR-146a in pancreatic cancer, whereby its reconstitution resulted in reductions of molecules such as EGFR, NF- $\kappa \mathrm{B}$ and IL-1 receptor-associated kinase 1 , leading to inhibition of invasive potential [34]. Restoration of miR-34, normally encoded by a p53-responsive

\section{Bibliography}

1. Jemal A, Siegel R, Ward E, Hao Y, Xu J, Thun MJ: Cancer statistics, 2009. CA Cancer J. Clin. 59(4), 225-249 (2009).

2. Wong HH, Lemoine NR: Pancreatic cancer: molecular pathogenesis and new therapeutic targets. Nat. Rev. Gastroenterol. Hepatol. 6(7), 412-422 (2009).

3. Moore MJ, Goldstein D, Hamm J et al.: Erlotinib plus gemcitabine compared with gemcitabine alone in patients with advanced pancreatic cancer: a Phase III trial of the National Cancer Institute of Canada Clinical Trials Group. J. Clin. Oncol. 25(15), 1960-1966 (2007).

4. Philip PA, Benedetti J, Fenoglio-Preiser C et al.: Phase III study of gemcitabine [G] plus cetuximab $[\mathrm{C}]$ versus gemcitabine in patients [pts] with locally advanced or metastatic pancreatic adenocarcinoma [PC]: SWOG S0205 study. J. Clin. Oncol. (Meeting Abstracts) 25(18 Suppl.), (2007)

(Abstract 4509).

5. Buanes T, Maurel J, Liauw W, Hebbar M, Nemunaitis J: A randomized Phase III study of gemcitabine $(G)$ versus GV1001 in

gene, has also been found to reduce the number of pancreatic cancer stem cells [35], which are thought to be responsible for disease relapse and resistance to chemotherapy and radiotherapy.

Despite the setbacks encountered with a number of targeted agents in pancreatic cancer, steady improvements have been achieved over the years in our understanding of the biology of pancreatic cancer and the development of novel therapeutic strategies. A number of genetically targeted agents have shown encouraging results in the laboratory, although a major obstacle would be the availability of an efficient and sustained delivery system in humans. In this aspect, the use of mesenchymal stem cells, which are bone marrow-derived nonhematopoietic precursor cells that are naturally attracted to the tumor site, is still under investigation but may hold promise in the future treatment of this devastating disease [36].

\section{Financial \& competing interests disclosure \\ The authors have no relevant affiliations or financial involvement with any organization or entity with a financial interest in or financial conflict with the sub- ject matter or materials discussed in the manuscript. This includes employment, consultancies, honoraria, stock ownership or options, expert testimony, grants or patents received or pending, or royalties. \\ No writing assistance was utilized in the production of this manuscript.}

sequential combination with $\mathrm{g}$ in patients with unresectable and metastatic pancreatic cancer (PC). J. Clin. Oncol. (Meeting Abstracts) 27(15 Suppl.), (2009) (Abstract 4601).

6. Olive KP, Jacobetz MA, Davidson CJ et al: Inhibition of Hedgehog signaling enhances delivery of chemotherapy in a mouse model of pancreatic cancer. Science 324(5933), 1457-1461 (2009).

7. Alberts SR, Foster NR, Morton RF et al.: PS-341 and gemcitabine in patients with metastatic pancreatic adenocarcinoma: a North Central Cancer Treatment Group (NCCTG) randomized Phase II study. Ann. Oncol. 16(10), 1654-1661 (2005).

8. Sloss CM, Wang F, Liu R et al:: Proteasome inhibition activates epidermal growth factor receptor (EGFR) and EGFR-independent mitogenic kinase signaling pathways in pancreatic cancer cells. Clin. Cancer Res. 14(16), 5116-5123 (2008).

9. Chuang HC, Kardosh A, Gaffney KJ, Petasis NA, Schonthal AH: COX-2 inhibition is neither necessary nor sufficient for celecoxib to suppress tumor cell proliferation and focus formation in vitro. Mol. Cancer 7, 38 (2008).
10. Jones S, Zhang X, Parsons DW et al.: Core signaling pathways in human pancreatic cancers revealed by global genomic analyses. Science 321(5897), 1801-1806 (2008).

11. Wong HH, Lemoine NR: Biological approaches to therapy of pancreatic cancer. Pancreatology 8(4-5), 431-461 (2008).

12. Chawla SP, Chua VS, Fernandez L et al.: Advanced Phase I/II studies of targeted gene delivery in vivo: intravenous Rexin-G for gemcitabine-resistant metastatic pancreatic cancer. Mol. Ther. 18(2), 435-441 (2010).

13. Mulvihill S, Warren R, Venook A et al:: Safety and feasibility of injection with an E1B-55 kDa gene-deleted, replicationselective adenovirus (ONYX-015) into primary carcinomas of the pancreas: a Phase I trial. Gene Ther. 8(4), 308-315 (2001).

14. Hecht JR, Bedford R, Abbruzzese JL et al.: A Phase I/II trial of intratumoral endoscopic ultrasound injection of ONYX-015 with intravenous gemcitabine in unresectable pancreatic carcinoma. Clin. Cancer Res. 9(2), 555-561 (2003). 
15. Wong HH, Lemoine NR, Wang Y: Oncolytic viruses for cancer therapy: overcoming the obstacles. Viruses 2(1), 78-106 (2010).

16. Wang Y, Gangeswaran R, Zhao X et al:: CEACAM6 attenuates adenovirus infection by antagonizing viral trafficking in cancer cells. J. Clin. Invest. 119 (6), 1604-1615 (2009).

17. Nishimoto T, Yoshida K, Miura Y et al: : Oncolytic virus therapy for pancreatic cancer using the adenovirus library displaying random peptides on the fiber knob. Gene Ther. 16(5), 669-680 (2009).

18. Hiley CT, Yuan M, Lemoine NR, Wang Y: Lister strain vaccinia virus, a potential therapeutic vector targeting hypoxic tumours. Gene Ther. 17(2), 281-287 (2010).

19. Tysome JR, Briat A, Alusi G et al.: Lister strain of vaccinia virus armed with endostatin-angiostatin fusion gene as a novel therapeutic agent for human pancreatic cancer. Gene Ther. 16(10), 1223-1233 (2009).

20. Hirano S, Etoh T, Okunaga R et al.: Reovirus inhibits the peritoneal dissemination of pancreatic cancer cells in an immunocompetent animal model. Oncol. Rep. 21(6), 1381-1384 (2009).

21. Zhang Z, Huang Y, Newman K et al.: Reexpression of human somatostatin receptor gene 2 gene mediated by oncolytic adenovirus increases antitumor activity of tumor necrosis factor-related apoptosis-inducing ligand against pancreatic cancer. Clin. Cancer Res. 15(16), 5154-5160 (2009).

22. Bortolanza S, Bunuales M, Otano I et al.: Treatment of pancreatic cancer with an oncolytic adenovirus expressing interleukin-12 in syrian hamsters. Mol. Ther. 17(4), 614-622 (2009).
23. Leitner S, Sweeney K, Oberg D et al.: Oncolytic adenoviral mutants with $E 1 B 19 K$ gene deletions enhance gemcitabine-induced apoptosis in pancreatic carcinoma cells and anti-tumor efficacy in vivo. Clin. Cancer Res. 15(5), 1730-1740 (2009).

24. Nelson AR, Davydova J, Curiel DT, Yamamoto M: Combination of conditionally replicative adenovirus and standard chemotherapies shows synergistic antitumor effect in pancreatic cancer. Cancer Sci. 100(11), 2181-2187 (2009).

25. Watanabe I, Kasuya H, Nomura N et al.: Effects of tumor selective replicationcompetent herpes viruses in combination with gemcitabine on pancreatic cancer. Cancer Chemother. Pharmacol. 61(5), 875-882 (2008).

26. Alberts SR, Schroeder M, Erlichman C et al:: Gemcitabine and ISIS-2503 for patients with locally advanced or metastatic pancreatic adenocarcinoma: a North Central Cancer Treatment Group Phase II trial. J. Clin. Oncol. 22(24), 4944-4950 (2004).

27. Rejiba S, Wack S, Aprahamian M, Hajri A: $K$-ras oncogene silencing strategy reduces tumor growth and enhances gemcitabine chemotherapy efficacy for pancreatic cancer treatment. Cancer Sci. 98(7), 1128-1136 (2007).

28. Chen C, Yu Z: siRNA targeting HIF-1 $\alpha$ induces apoptosis of pancreatic cancer cells through NF- $\kappa \mathrm{B}$-independent and -dependent pathways under hypoxic conditions. Anticancer Res. 29(4), 1367-1372 (2009).

29. Li M, Zhang Y, Bharadwaj U et al.: Down-regulation of ZIP4 by RNA interference inhibits pancreatic cancer growth and increases the survival of nude mice with pancreatic cancer xenografts. Clin. Cancer Res. 15(19), 5993-6001 (2009).
30. Li M, Zhang Y, Liu Z et al.: Aberrant expression of zinc transporter ZIP4 (SLC39A4) significantly contributes to human pancreatic cancer pathogenesis and progression. Proc. Natl Acad. Sci. USA 104(47), 18636-18641 (2007).

31. Wang Z, Zhang Y, Li Y, Banerjee S, Liao J, Sarkar FH: Down-regulation of notch-1 contributes to cell growth inhibition and apoptosis in pancreatic cancer cells. Mol. Cancer Ther. 5(3), 483-493 (2006).

32. Yang G, Huang C, Cao J, Huang KJ, Jiang T, Qiu ZJ: Lentivirus-mediated shRNA interference targeting STAT3 inhibits human pancreatic cancer cell invasion. World J. Gastroenterol. 15(30), 3757-3766 (2009).

33. Lee EJ, Gusev Y, Jiang J et al: : Expression profiling identifies microRNA signature in pancreatic cancer. Int. J. Cancer 120(5), 1046-1054 (2007).

34. Li Y, Vandenboom TG 2nd, Wang Z et al.: miR-146a suppresses invasion of pancreatic cancer cells. Cancer Res. 70 (4), 1486-1495 (2010).

35. Ji Q, Hao X, Zhang M et al.: MicroRNA miR-34 inhibits human pancreatic cancer tumor-initiating cells. PLoS ONE 4(8), E6816 (2009).

36. Kallifatidis G, Beckermann BM, Groth A et al: : Improved lentiviral transduction of human mesenchymal stem cells for therapeutic intervention in pancreatic cancer. Cancer Gene Ther. 15(4), 231-240 (2008). 